\title{
ARTIFICIAL IMPREGNATION: ESSAYS IN TUBAL INSEMINATION*
}

\author{
By Robert L. Dickinson, M.D., New York, N. Y.
}

$\mathrm{T}$ HE only unimpugnable evidence of efficacious instrumental insemination comes from our successful and scientific brother the veterinarian, because he can exercise complete supervision over his patient. Human tests are blocked by aversions, vitiated by reticences, and happy results are not susceptible of rigid proof, because intercourse may have followed treatment. The plausibility of any claim must rest on the publication of histories and methods in some detail. All attempts should be listed, inasmuch as this is one of the clinical series in which no one observer is able to present any large group of cases. The records will follow in a later article. In this paper the technic is reported on, with a summary of results.

As marriage is an experiment in procreation without preliminary tests for fitness or instruction in method, it should be considered salisfactory that there occur something like 90 per cent of successes-although some cities and countries average 20 per cent of childless marriages. Inability to conceive at all accounts for about half the "sterile" marriages (Schaeffer). The husband's defective semen, judged mostly from a single examination of a condom specimen, is generally said to be responsible for one in five of these disabilities, but Huhner doubles this particular figure, and I also would place it higher. We have then to consider those couples where the semen is vigorous and there is no obstruction in the lower genital passages, and no infection. Even where an anteflexion or retroversion is present, before operation is considered the woman is entitled to any measure that holds out a reasonable promise. Suppose this method of artificial insemination does hold out a reasonable promise. Then the program will be as follows. Man and wife are each brought up to good physical condition and their sex-life is studied and regulated. These measures failing, trial will be made of a course of instrumental impregnation. The presence of marked flexion or mobile retroversion need not preclude this, since safe passage through the dangers of the miscarriage period can usually be accomplished with a sufficient degree of care. The instrumental instillation failing, operation may be done for well marked anteflexion or retroversio-flexion, particularly in the presence of real pain or disability. Laparotomy on the single indication of sealed tubes to be opened or deployed is generally frowned upon because of a common agreement in the reports of scant success. Laparotomy to study and incise the ovaries, where it is supposed ova need freeing, has an advocate in Reynolds. Laparotomy after six months of marriage just to find out what can be the matter with a woman who appears to be sound has been urged only by Solomons of Dublin. Whatever the program that appeals to any student of the question, there will hardly be denial that better knowledge is needed concerning what constitutes normal sex life, concerning the male secretion, genital incompatabilities, and physiologic deficiencies.

*Read at the Forty-fifth Annual Meeting of the American Gynecological Society, Chicago, May 26, 1920 . 
Nor can some or all of the following considerations be passed by in any particular problem under review.

\section{PRELIMINARIES}

Verification of the male secretion will one day be the routine first step. Two or three microscopic examinations are needed. Semen does not run true to form. It is a gauge very sensitive to changes in general physical condition and may present quick and sweeping alterations without apparent adequate reason. Activity of spermatozoa is of major importance, but survival-hours are all-important too. Stated in the baldest terms, the two requisites are that in the fresh warm specimen seen in the deep covered slide speedy transits across several fields should be under way, and, furthermore, at room temperature, activity should persist for hours. One may see the trapped tail, the feeble stroke, short lived action, or even azoospermia yield to a vacation or reduction of obesity, and such betterment, combined with cure of the commonest sterility lesion in women, endotrachelitis, sufficient to start a family. Coitus is to be regulated. Prolonged intervals may produce as poor specimens as undue frequency. An individual has a normal cycle which will deliver the best result, and this might be worked out, but 7 to 10 days is a fair average for the liveliest persistence in my few studies of multiple specimens.

On the part of the woman, correction of flabby or rapid obesity counts, as does general condition, good periods, and sex responsiveness.

Lcucorrhea of an acid or purulent character is to be arrested, and this goes hand in hand with its main cause, unhealthy condition of the lining of the cervix. The spectacular cures are those in which healing of the raw surface or drainage of the clogged canal is instantly followed by conception.

The mild alkaline douche an hour or so before coitus is in use for strongly acid vaginal secretions and will be used even where litmus does not make any such accusation. I cannot get a chemist to devise any simple quantitative test of vaginal acidity.

Study of the reaction of a vaginal secretion on a particular semen is of limited application for most doctors in office work, and tests carried up into the cavity of the uterus are subject to too many errors to belong to any but a few experimenters. Some of these moot points are touched on in the second portion of this paper.

Retention of semen by a condom-covered tampon, inserted at once after emission, may be tried where the vulva gapes, but my various experimental modifications of pessaries to develop a semen trap have not been effective.

Tests of the patency of the tubes will be routine when the procedure is standardized. I tried Cary's injection of silver salts, with vague $x$-ray shadows. Cary's simple instillation of sterile fluid in the genupectoral posture is promising. If it disappears in quantities over 10 minims the way should be clear. My trials that show free passage of semen have demonstrated patency in the same way. These procedures are infinitely easier though less certain than the injection of oxygen and its appearance under the diaphragm-calling as oxygen does for apparatus and special skill and with sequelæ sometimes distressing. 
Lastly we rule out of consideration patients with gonorrhea or suspicion thereof, tubal distention or tenderness and inflammatory processes of any degree in the pelvis.

\section{TECHNIC}

During the office examination one selects the shortest or smallest bivalve speculum that will make a good exhibit of this particular cervix in the kneechest position, and also tests a snug fitting curved pipette in the internal os. A note or mark is made on the pipette to show how far up 10 minims will fill its caliber. After a date is made-to follow a week of continence-the husband is given a sterile test tube, dry and corked. He is directed to wash earefully and secure a friction specimen about an hour before the appointed time, taking care that the inside of the cork makes no contacts, and to keep the tube warm but not hot, under a warm water bag or in a Thermos bottle. He is to verify by telephone a successful production.

The following are sterilized: bivalve speculum, single tenaculum, two or three pipettes in test tubes, applicators, cotton-tipped, and towel and tray on which to lay out the above. (Forceps and scissors in case condom specimen is to be used). All pipettes to be dried.

At the home some third person is to be near at hand, though not necessarily in sight. Good illumination is needed--droplight and head mirror or headlight preferred. Bladder and bowel are previously emptied. All the materials are spread conveniently at hand. The patient takes a real knee-chest posture at the edge of and across the bed. The bivalve gives a clear view of the ballooned vagina, and also a free play to the uterus such as cannot oblain with this instrument in the dorsal posture. This free play is important in gaining ready access to the cavity of the uterus. The tenaculum steadies the cervix and serves to draw open the canal, which should rarely need to be wiped and on which no antiseptic should be used. The pipette is now very gently filled above a point known to be 10 minims. (The uterus holds 8 to 10 minims.) The tip touches first the interior of the cervical canal as high as may be and is passed to near the fundus. The fit to the internal os prevents regurgitation, or if it does not, one makes a change. Gentle, steady pressure is made on the bulb until "unwell feelings" are produced and continued till there is consciousness of slight distress in the sides of the abdomen low down, at which time the Fallopian tubes are presumed to have fluid in them.

Then pipette, tenaculum and speculum are withdrawn, the patient slides onto her side with the hips a little elevated, to remain thus at least an hour.

The Skene uterine pipette is a bit thicker than a uterine sound and curved like the sound. Its opening must not be minute, as Huhner's curled up forms of defunct spermatozoa are produced by quick suction through narrow orifices. The advantage over any syringe is that the contents are all in sight, the interior is easily eleaned and various sizes are at hand. The sharply recurved single hook of Emmet does not give the discomfort of the heavy double forms.

The following are variants in the above methods. As a substitute for ejaculation into the test tube one is reluctant to concede the use of the condom, as it presents mahy more possibilities of contamination. The vulva and the 


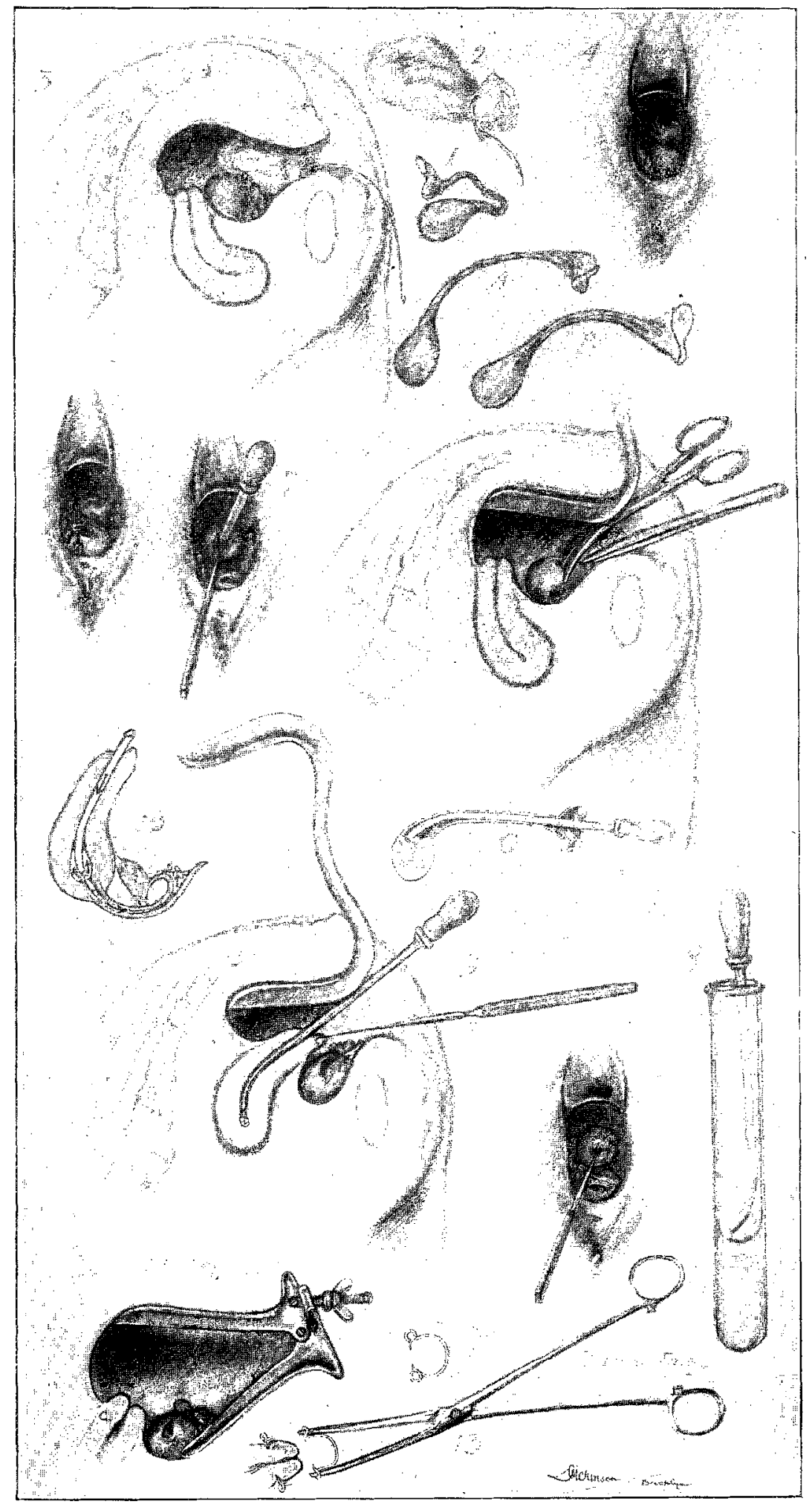

Fio. 1-Methods on trial in past years. Part of author's exhibit at the International Congress in London in 1913. The condom as the reservoir, pipette instillation, clip closure of cervix. I. The neck of the condom twisted, after ejaculation, without removal from the vagina. 2. The tampon that is introduccd before rising to hold the condom in place in order that the patient may come to the offire. 3 . The patient in the knee-chest posture. 4. The spectlum in place. 5. The cover steadied with a forceps and slit, as seen in 6,7 and 8 . The Shene pipette, sterile dry and warm, sucks from the slit and injects as shown in into or through the tube, as ro gives it diagrammatically; $I I$ is ready for the clip. IZ, placed by a Bozeman forceps as seen in $I 3$, before and after placing. Alternate plans are given in $A$, and $B$ the by a Bozeman forceps as seen in $I 3$, before and after placing. Alternate plans are given in $A$, and $B$ the
condom uncoiled with its head still in the vagina, $C$, the pipette passed inlo it; findly $D$, employing a bivalve in lieu of a Sims speculum when one dispenses with a nurse. 
penis are washed, and coitus occurs with the condom. The contents may be kept warm in two ways. One way is to tie the mouth and drop the condom into a test tube which is laid under a warm water bag. The other takes care to leave the cover and its contents within the vagina, twisting the part hanging out and returning this part inside the passage. Under these circumstances there can be no danger of chilling or overheating and thus damaging the specimen. When ready to inject, the patient having taken the knee-chest posture and the speculum being in place, the condom is steadied with forceps and slit with scissors to let the pipette suck out the amount needed. When insemination is done at the office this has been the usual method, the condom having been retained by a tampon furnished to and placed by the patient. Finally it may be noted that one genitourinary specialist obtains a fresh specimen for injection by providing facilities for coitus at the office.

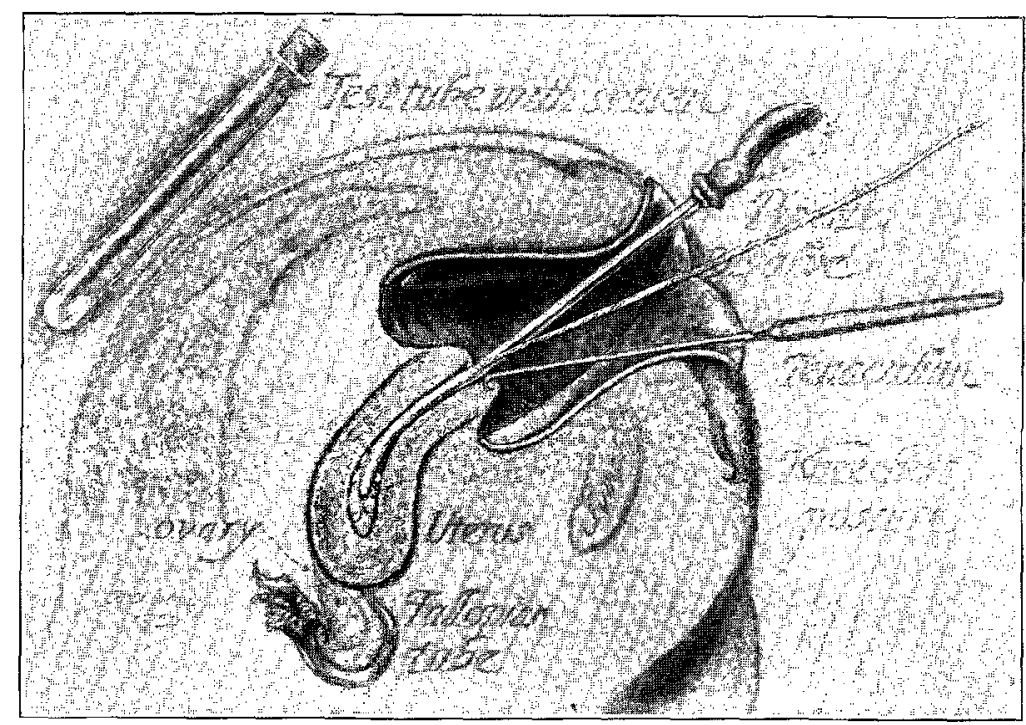

Fig. 2.-Insemination, using a short bivalve speculum, a single, well-angulated tenaculum; a pipette with the curve of a uterine sound and of a size to fit the internal os, and a large rubber butb. The body of the uterus is shown distended with semen.

Where autoerotic or cover processes completely inhibit, a specimen may be obtained by slipping the mouth of a sterile test tube within the opened labia immediately upon withdrawal. Washing of the genitals of both should have preceded this also. In place of the test tube one of the little spherical soft rubber bulbs with the soft tip has been used. These devices are worth remembering for another purpose, moreover. By such methods of collection semen can be secured in quantity in those instances where a man avers that he is desirous of having chiidren but refuses to take the steps necessary to prove that the default is not his. Some such plan is necessary when he asks for or consents to operation on his wife but declines an essential preliminary, since no surgeon nowadays is excusable for any operative measure or any gynecologist for a course of treatment on a woman for sterility until a good quality of male product can be certified. Finally, this product must be shown 
to be of good quality at the time and not merely to have been right in some past period.

\section{OBJECTIONS}

1. The possibility of infection of tube and peritoneum cannot be excluded. But normal insemination involves something traveling an inch an hour from the vagina-after labial and subpreputial "smears"-up into the uterus or tube or peritoneum, and all canals exhibit some reverse currents. If we use semen that has spurted clean into a sterile test tube and the cork is safeguarded, a specimen above reproach seems to be furnished. To be sure material from the canal of the cervix may be carried on the tip of the pipette and thrown into the tube and reach the peritoneum. (Curtis has demonstrated bacterial travels.) But in the absence of gonorrbea, of mucopurulent leucorrhea or cervical catarrh, production of salpingitis or peritonitis seems to the writer unlikely with a good technic. So far as symptoms go I am able to report that mong 31 women none developed uterine colic, one with a half-hour tubal colic, and one in bed several days with discomfort, but without exudate or tender tube.

2. Old tubal disease may be lighted up anew. It may, for a tubo-uterine orifice never closes. For the present at least such cases should be avoided.

3. It is possible that all that injection of semen accomplishes is to open the tube, in which case other fluids would do as well, and antiseptics be safer as used by Stone and Boveé. Only tests can tell.

4. One can have no assurance that coitus subsequent to the treatment was not the real agent in procuring conception. This may be true and this is the reason that the veterinary surgeon can offer proofs which we cannot.

5. It fails with semen which is not vigorous. It was devised for just such cases and it has not helped so far.

6 . The field is very limited because patients revolt at the idea, or give it one trial instead of half a dozen. They prefer laparotomy. All this is exact, but it does not relieve us of the responsibility of preventing the patient taking the greater risk should there be a simpler way, did we but have the will to face the distastefulness of working it out.

\section{UTERINE INSTILLATTON}

Uterine instillation is insufficient. The material injected into the cavity of the body of the uterus seemed to drain out promptly. This is probably because of the intermittent contractions normal and constant in nonpregnant uteri, and because a degree of opening up of the internal os has occurred in making the deposit. To overcome this, vaginal tampons were tried and abandoned. A clip was built to snap on the cervix and prevent exit of the semen, but it was not effective. Therefore the deeper placing of the seed was undertaken. All the good results have been obtained since this time.

In so far as repetition of the procedure is concerned, three attempts at monthly intervals should be the minimum and six should be asked, explaining that six months is not an unusual time for normal means in normal people. As to the time of the month, three of our conceptions followed injection within a week of the expected period, the period coming on incompletely. 


\section{SUMIMARY}

In women presenting histories or pelvic findings pointing to the sealed tube following milder types of salpingitis, entirely quiescent, injection into the uterine cavity of active semen produced no results in twelve instances. Strong pressure was not deemed warranted.

In women with no gonorrheal histories or findings, free from cervical inflammations and evident uterine, tubal or ovarian lesions or abnormalities, living semen of the poorer grades produced no results, in nineteen patients. No infection followed except in one possible instance and that of mild type. Several of these received three trials.

With fairly normal pelvic organs and semen of good quality, five pregnancies followed and are believed to have been due to tubal insemination. The kneechest posture, the curved pipette fitting the internal os and carried nearly to the fundus, injection into the tubes, horizontal rest, and repetition three to six times--these are considered important. Trial of this method may well precede resort to operation-save those done for external obstructions.

438 West ONe Hundred SiXteenti Street.

(For aiscussion, see $p .306$. ) 